Revue internationale P.M.E.

Économie et gestion de la petite et moyenne entreprise

\title{
Logique économique et logique sociale : La double rationalité de l'entreprise informelle et ses implications en matière de financement
}

\section{Bernard Haudeville}

Volume 5, numéro 3-4, 1992

URI : https://id.erudit.org/iderudit/1008152ar

DOI : https://doi.org/10.7202/1008152ar

Aller au sommaire du numéro

Éditeur(s)

Presses de l’Université du Québec

ISSN

0776-5436 (imprimé)

1918-9699 (numérique)

Découvrir la revue

Citer cet article

Haudeville, B. (1992). Logique économique et logique sociale : La double rationalité de l'entreprise informelle et ses implications en matière de financement. Revue internationale P.M.E., 5(3-4), 13-20.

https://doi.org/10.7202/1008152ar
Résumé de l'article

La structure productive des pays en voie de développement montre l'importance des entreprises informelles. C'est le cas en particulier en Afrique où elles occupent une large fraction de la population. Ces entreprises se caractérisent, entre autres, par la non-distinction de l'activité productive et de l'activité sociale du ménage-entrepreneur. De ce fait, le principe d'autonomisation de la sphère des activités économiques qui fonde l'économique en tant que science, n'a pas d'application ici. Les critères habituels de la rationalité économique ne peuvent plus servir de guide dans l'analyse des risques et des rendements de différents projets, ce qui rend sans intérêt les méthodes d'évaluation employées par les institutions financières. 


\title{
Logique économique et logique sociale : La double rationalité de l'entreprise informelle et ses implications en matière de financement
}

\author{
Bernard HAUDEVILLE* \\ Université du Maine et \\ Institut Orléanais de Finance
}

\begin{abstract}
RÉSUMÉ
La structure productive des pays en voie de développement montre l'importance des entreprises informelles. C'est le cas en particulier en Afrique où elles occupent une large fraction de la population. Ces entreprises se caractérisent, entre autres, par la non-distinction de l'activité productive et de l'activité sociale du ménage-entrepreneur. De ce fait, le principe d'autonomisation de la sphère des activités économiques qui fonde l'économique en tant que science, n'a pas d'application ici. Les critères habituels de la rationalité économique ne peuvent plus servir de guide dans l'analyse des risques et des rendements de différents projets, ce qui rend sans intérêt les méthodes d'évaluation employées par les institutions financieres.
\end{abstract}

\begin{abstract}
Informal sector industries accounts for a noticeable proportion of GNP in developing countries, especially in Africa. Among other distinctive features, informal businesses are characterized by the lack of a clear-cut difference between their productive and social activities. Therefore a fundamental constituant of economic theory is missing and standard economic principles cannot fully apply. In particular, the respective risks and rewards of projects cannot be assessed in the light of the usual rational economic criteria, nor can financial institutions base their decisions on standard methods of rating.
\end{abstract}

* Bernard Haudeville est docteur ès sciences économiques de l'Université de Paris I et licencié ès lettres. Agrégé de sciences économiques et professeur à l'Université du Maine, il dirige le Département d'économie et de gestion à la Faculté de droit et des sciences économiques du Mans, après avoir enseigné aux Universités d'Abidjan (1977-1978) et de Niamey (1984-1988). Adresse : 54, rue Alexandre Dumas, 45100 Orléans, France. 


\section{RESUMEN}

La estructura productiva de los países en vías de desarello muestra la importancia de las empresas informales. Es el caso de Africa, donde ocupan una franja importance de la población. Estas empresas se caracterizan por la no districión de la actividad productiva y de la actividad social del pequeño empresario. Debido a esto, el principio de autonomía de las actividades económicas que se basa en lo económica en tanto que ciencia no tiene aqui aplicación. Los criterios habituales de la racionalidad económica no peuden servir de guia en el análisis de los riesgos y de los rendionientos de la diferentes proyectos, por lo que los métodos de evaluación empleados por las instituciónes financieras son, en este caso, inútiles.

\section{Introduction}

L'activité de production de biens et services dans les pays en voie de développement correspond, comme ailleurs, à différents types juridiques et différentes classes de taille pour les entreprises. Toutefois, l'organisation du tissu productif présente dans ces pays quelques caractéristiques distinctives. Si les grandes entreprises, et les très grandes, n'y sont pas rares, les entreprises de taille intermédiaire, moyennes ou moyennes grandes, qui constituent l'essentiel de la population, dans certains pays développés comme l'Allemagne, à la fois en nombre et en production, y sont très peu nombreuses. On trouve par contre un très grand nombre de petites et très petites entreprises, souvent réduites à la seule personne de l'entrepreneur, dépassant rarement quelques salariés et apprentis. Parmi les petites et très petites entreprises, certaines ont une existence légale et déclarée tandis que d'autres sont rangées dans ce qu'il est convenu d'appeler le secteur informel. Les PME dans les pays en voie de développement, ce sont donc quelques entreprises de taille moyenne et une nébuleuse de petites organisations où prédominent les entreprises informelles. Que l'on mesure par le nombre d'entreprises, l'emploi fourni ou les revenus créés, la catégorie informelle est le plus souvent majoritaire. Cela est en particulier le cas dans la plupart des pays d'Afrique francophone et sans doute dans de nombreux pays du continent africain en général. Pour cette raison, cette catégorie est parfois considérée comme un «gisement » d'entreprises au sens formel du terme, ce qui est sans doute faux. Mais même si sa vocation est de demeurer informelle, l'importance du phénomène justifie amplement l'intérêt qui y est porté.

Dans ce texte, on s'intéressera essentiellement aux petites et très petites entreprises et à leurs modes de financement. On commencera par une présentation du cadre dans lequel s'inscrit le fonctionnement de ce type d'entreprise avant d'en tirer les conséquences concernant les modes de financement possibles 
pour ce genre d'activité. On terminera par quelques remarques sur l'évolution des politiques de développement.

\section{Rationalité économique et statut de l'entreprise informelle}

Comme cela a été largement exposé par Karl Polanyi et Louis Dumont entre autres ${ }^{1}$, l'émergence d'un concept d'entreprise résulte de l'autonomisation de l'économique par rapport au social. L' «entreprise » n'est en fait qu'un démembrement de l'individu dans sa fonction économique, l'autre partie étant constituée par le «consommateur ». Même si cette abstraction s'est révélée particulièrement féconde dans l'analyse économique, elle conserve cependant un caractère d'artifice qui apparaît dans certains aspects de la science économique moderne. La comptabilité nationale permet facilement de mettre en lumière certains de ces problèmes. Le traitement de l'entrepreneur individuel est sans doute le plus révélateur. Dans l'ancien système français, par exemple, les ménages étaient artificiellement scindés. La partie production de l'entrepreneur individuel était agrégée avec les entreprises non financières. Le revenu de l'entrepreneur individuel était ensuite versé et affecté au ménage pour ses opérations de dépense et d'épargne ${ }^{2}$. Aucune épargne n'apparaissait dans l'entreprise individuelle, mais le ménage contribuait au financement des investissements de l'entreprise par une opération portée en ressource du compte de capital des entreprises non financières (financement des investissements par les entrepreneurs individuels). Le nouveau système adopté en 1976, conforme au système normalisé des Nations Unies, traite désormais de façon unique l'entrepreneur individuel comme un ménage, ce qui conduit à faire apparaître un compte de production et un compte d'exploitation dans un secteur institutionnel dont la principale opération économique est... la consommation ${ }^{3}$. Le traitement de certains biens est aussi fonction de la nature de l'agent qui les acquiert. Une automobile achetée par une société ou quasi-société constitue une opération d'investissement enregistrée en FBCF au compte de capital. La même automobile achetée par un ménage constitue une consommation enregistrée au compte

1. K. Polanyi, La grande transformation, Paris, éd. Gallimard, 1983 ; L. Dumont, Homo Aequalis, Paris, Gallimard, 1977. Le rapprochement de ces deux auteurs, l'un ethnologue, l'autre sociologue, ne va pas sans une certaine dose d'arbritraire. Mais ce qu'ils ont en commun, et ce qui les distingue des autres, à savoir l'accent mis sur le caractère construit par opposition à «naturel » de la réalité économique, est pour notre propos plus important que ce qui les sépare.

2. J. Marchal, Cours d'Économie politique, Paris, Ed. Genin, 1957, Titre 1, chap. 11.

3. J.P. Piriou, La Comptabilité nationale, éd. La Découverte, 1987. 
d'utilisation du revenu. On peut remarquer que, dans son traitement de la consommation, M. Friedman ne prend en compte que les services consommés pendant la période considérée, les biens durables étant simplement producteurs de services, ce qui rejoint par conséquent un traitement en termes de biens de capital des biens durables ${ }^{4}$.

Les remarques précédentes montrent bien qu'une même opération peut obéir à des logiques différentes selon le cadre dans lequel elle s'inscrit. En effet, les éléments et les procédures conduisant un ménage à la décision d'acheter un bien durable différeraient le plus souvent des éléments et procédures utilisées par une entreprise pour la même dépense. Si l'on prend comme exemple le marché des véhicules de tourisme, il est douteux que les ménages effectuent les calculs de coût au kilomètre ou de rentabilité de l'investissement. Par contre l'esthétique ou l'image, associée à tel ou tel modèle, auront pour eux davantage d'importance. On peut du reste constater qu'il existe des segments de marché spécialisés pour les voitures d'affaire ou d'entreprise. Ainsi, il est évident que l'achat d'un bien qui peut constituer un bien de capital, n'implique pas de l'acquéreur l'insertion dans une logique de rentabilisation de ce bien. De la même façon, le fait de produire des biens et des services destinés à la vente ne requiert pas non plus forcément la mise en œuvre d'une rationalité de type entrepreneurial. C'est ce que nous voulons essayer de mettre en évidence pour l'entreprise informelle ou plus généralement pour la petite entreprise dans les PVD. Il n'est en effet pas toujours aisé de tracer des frontières dans la catégorie des petites entreprises entre celles qui sont officielles et celles qui sont informelles. Les critères usuels tels que l'enregistrement, l'existence d'un emplacement fixe, l'autorisation d'exercice ne sont pas sans ambiguïtés. L'exemple d'Abidjan à la période actuelle illustre bien ce problème $e^{6}$.

Ce qui caractérise la petite entreprise en particulier en Afrique, c'est précisément le fait que l'activité de production et l'activité du ménage sont indissolublement liées. Cela n'aurait aucun sens de tenter ici les dissociations faites par la comptabilité nationale et qui ont été rappelées ci-dessus. C'est directement l'homme dans sa dimension sociale, c'est-à-dire totale, par opposition au réductionnisme de l'homo economicus, qui est pris en compte ici. De ce fait, la rationalité mise en œuvre ne peut se réduire aux éléments habituels du

4. M. Friedman, $A$ Theory of the Consumption Function, Princeton et NBER, 1957.

5. Réf. G. Nihan, « Le secteur non structuré, signification, aire d'extension du concept et application expérimentale ", Tiers Monde, 1980, n 82 ; M.P. Van Dijk, Burkina Faso, le secteur informel de Ouagadougou, Paris, L'Harmattan, 1986.

6. $\mathrm{Ph}$. Haeringer, « Ni involutive ni accumulatrice, la reproduction évolutive de l'artisanat innovateur dans une métropole africaine », dans J.L. Lespès, Les pratiques juridiques, économiques et sociales informelles, Paris, PUF, 1991. 
calcul économique. On sait par exemple que, dans la plupart des cas, la petite entreprise n'a pas de stocks et que la production est davantage commandée par les besoins de dépense du ménage que par une quelconque stratégie vis-à-vis du marché. Le tailleur ou le tâcheron redouble d'effort dans la perspective d'un achat ou d'une dépense liée à une fête ou à une cérémonie et non en réponse à l'évolution du prix de leurs services. L'absence généralisée de comptabilité et la seule prise en compte des coûts directs des matériaux vont évidemment dans le même sens. De même, si l'on a pu dire que le secteur informel maximise la substitution du travail au capital, cela souligne bien le lien direct qui s'établit entre la production et la création de revenus ${ }^{7}$. La petite entreprise, c'est essentiellement le moyen de se procurer un revenu pour toute une fraction, nombreuse, de la population que le secteur moderne ne peut absorber, voire rejette. Là encore, l'exemple d'Abidjan à l'heure actuelle est parfaitement explicite. Mais c'est aussi un mode de produire qui répond bien à l'attente de la fraction la plus démunie de la population qui en constitue la clientèle et surtout s'insère parfaitement dans le réseau des relations sociales de la communauté, géographique ou ethnique, d'appartenance. Cette insertion est d'une certaine façon source de contraintes et enserre l'activité de l'entreprise dans un ensemble d'interdits et d'obligations coutumières qui peuvent être ressenties comme autant d'obstacles à la maximisation d'une quelconque fonction objectif. Mais d'un autre côté, elle a valeur d'assurance par les solidarités qu'elle entraîne. Aussi faudrait-il en toute logique considérer le réseau de relations sociales comme un actif incorporel...

Dans la mesure où, comme le note J.L. Lespès, dans la plupart des PVD, la société ancienne fondée sur l'agriculture et les liens de parenté n'a pas entièrement été remplacée par la société marchande généralisée, le secteur informel joue un rôle d'amortissement ou d'accompagnement des transformations de la société 8 . Il a de ce fait un rôle protecteur pour les individus et c'est sa fonction principale ${ }^{9}$. Il produit de la sécurité pour des individus pratiquement sans ressources, et c'est dans ces termes que doit s'apprécier l'efficacité de cette forme particulière d'organisation. On est bien loin des critères économiques habituels d'appréciation et il ne faut donc pas s'étonner de la coupure avec la partie formelle ou moderne de l'économie qui, elle, raisonne en termes purement

7. Cl. de Miras, «Le secteur de subsistance dans les branches de production à Abidjan », Tiers Monde, 1980, vol. XXI, $\mathrm{n}^{\circ} 82$.

8. Réf. J.L. Lespès, « Les informalités tontinières : traditions et innovations », dans $M$. Lelart, La tontine : pratiques d'épargne informelle dans les pays en voie de développement, Paris, John Libbey, 1990.

9. B. Haudeville, «Épargne informelle et financement de l'entreprise productive », dans Hénault et M'Rabet (éd.), L'entrepreneuriat en Afrique francophone, culture, financement et développement, Paris, John Libbey, 1990. 
monétaires dans une logique économique standard ${ }^{10}$. Cette coupure apparaît en particulier au niveau du financement comme on va le montrer dans la deuxième partie.

\section{Critères d'efficacité et sources de financement}

Les éléments extra-économiques largement dominants qui viennent d'être rappelés rendent évidemment très difficile, voire impossible, une analyse financière au sens courant. De ce fait, il n'y a aucun critère objectif d'évaluation du risque et de la rentabilité du placement dans ce type d'entreprise. Il est logiquement normal que les financements obtenus du secteur formel et principalement des banques aient un caractère exceptionnel. L'absence quasi générale d'actif transférable pouvant servir de garantie est à elle seule dissuasive. Or le principal actif de la petite entreprise, c'est la solidarité dont le groupe fera preuve envers son titulaire en cas de besoin et ce n'est pas précisément un actif transférable à un créancier. Force est donc de se retourner vers les autres formes de financement : apport personnel, prêts familiaux, usure ${ }^{11}$, tontines. Le lien entre activité de production informelle et activité de finance informelle s'établit donc en premier lieu par défaut. Mais les caractéristiques structurelles communes à ces deux formes d'activité sont suffisamment fortes pour qu'on puisse parler d'une véritable congruence.

L'absence de critère d'évaluation du risque peut encore s'interpréter comme l'impossibilité de prendre en compte la dimension temporelle, ce qui correspond à une préférence quasi absolue pour le présent ${ }^{12}$. Dès lors, il convient de mettre l'accent sur les flux financiers, c'est-à-dire la trésorerie essentiellement. Il n'y a pas lieu de raisonner en termes de stocks et d'accumulation, mais d'entrées et de sorties de fonds qui constituent autant de recettes et dépenses pour le ménage. De ce fait, la durée d'immobilisation des fonds est courte, ce qui rend possible l'utilisation de certaines modalités de collecte comme la tontine.

10. Ce qui peut avoir des conséquences sur les comportements des individus dans la mesure où certains sont en contact avec les deux logiques.

11. Bien qu'on ait peu d'informations sur l'usure en raison de sa nature même, il semble bien qu'elle soit partout présente dans les PVD et sans doute pour une fraction importante des besoins de financement des petites entreprises.

12. Réf. E. Bloy et $\mathrm{Cl}$. Dupuy, «Adaptation des règles de gestion aux contraintes du financement informel », dans Hénault et M'Rabet (éd), L'entrepreneuriat en Afrique francophone, culture, financement et développement, Paris, John Libbey, 1990. 
Plus fondamentalement, l'esprit et les modalités particulières de fonctionnement des associations d'épargne et de crédit les rendent particulièrement aptes à participer au financement de la petite entreprise, qu'elle soit formelle ou informelle. Comme le note $\mathrm{H}$. Desroche ${ }^{13}$, le fondement de ces associations est l'entraide et la solidarité. Leur généralisation à l'ensemble des régions du monde, pays développés compris, et à presque toutes les époques de l'histoire, dit assez l'universalité de ces institutions. Nous sommes dans l'économie du don et du contre-don selon $\mathrm{H}$. Desroche et non dans l'économie d'échange. On retrouve la classification de Polanyi distinguant trois principes généraux d'organisation des systèmes sociaux: le système de marché, le système de redistribution et le système de réciprocité ${ }^{14}$. C'est ce dernier, fondé sur le don et le contre-don, qui caractérise le mieux les segments traditionnels des sociétés des PVD. Selon la définition, «tous les échanges ont un caractère gratuit et on s'attend à ce qu'ils soient payés de retour mais pas forcément par le même individu $»^{15}$. Ce système suppose une grande cohérence et une forte pression sociale pour assurer la crédibilité de cette réciprocité. Le passage à l'économie marchande généralisée s'accompagne de la disparition de cette pression et de la dissolution des liens entre individus qui étaient nécessaires pour la maintenir.

Si les associations d'épargne, comme les tontines par exemple, font pleinement partie dans leur plus grande majorité de ce système d'entraide et de réciprocité ${ }^{16}$, les petites entreprises, dans la mesure où elles débordent du comportement économique standard, et dans cette mesure précisément, se rattachent à la même logique. Ce qui distingue la "petite entreprise » dans son comportement, de l'entreprise tout court, c'est ce qui la rattache à cette économie de la réciprocité. Il y a donc véritablement symbiose entre la petite entreprise et l'épargne informelle dans la forme tontinière, qui va au-delà de la nécessité d'assurer le financement de la petite entreprise par d'autres moyens que les moyens formels classiques auxquels elle n'a pas accès.

Il est donc tout à fait nécessaire de considérer ensemble le secteur de la petite entreprise ou de l'entreprise informelle et l'activité des circuits d'épargne informelle. Le formidable renouveau d'intérêt pour ce domaine, auquel on assiste de la part des organisations non gouvernementales, intervenant dans le domaine du développement, résulte dans une large mesure de l'échec des

13. H. Desroche, «Des tontines Nord aux tontines Sud», dans M. Lelart, La tontine, Paris, éd. John Libbey, 1990.

14. J.L. Lespès, "Les informalités tontinières : tradition et innovation », dans Michel Lelart, La tontine, Paris, Éd. John Libbey, 1990.

15. K. Polanyi, La grande transformation, op. cit., p. 76.

16. Sauf peut-être certaines tontines dites financières qui finissent par avoir vis-à-vis de personnes n'appartenant pas au groupe une activité importante de prêt. 
politiques précédentes orientées vers la constitution de secteurs modernes puissants et capables d'exercer des effets d'entraînement. Il porte en lui l'amorce d'un renouvellement des politiques de développement faisant une plus large place à la plus grande partie de la population.

Il est sans doute illusoire cependant de placer trop d'espoir dans le développement du secteur informel. En effet, celui-ci fonctionne dans une optique de reproduction simple. Il ne réalise pas, ou très peu, d'accumulation. Or, le développement suppose que l'on passe à un schéma de reproduction élargie, ce que ne peut faire le secteur informel. Les petites entreprises existent même si elles ne sont pas appelées à grandir, comme le suppose la thèse de la transition qui en fait des PME potentielles. Elles fournissent emplois et revenus, ainsi que biens et services à faible coût. Certaines études montrent que les besoins en investissement dans ce secteur sont faibles et qu'il n'est guère souhaitable d'y accroître les immobilisations, la productivité du capital, mais aussi celle du travail tendant à baisser lorsque la taille de l'entreprise informelle, mesurée par son capital, augmente ${ }^{17}$ ou lorsque l'intensité en capital du processus de production augmente ${ }^{18}$. D'un autre côté, il y a une épargne collectée par des circuits de financement informels dont on ne mesure pas très précisément l'importance, variable sans doute selon les régions et les pays, mais qui n'est jamais négligeable surtout si on la compare avec le volume des financements distribués par les canaux officiels. D'où l'idée, sur laquelle nous réfléchissons depuis quelques années maintenant dans le cadre de l'UREF, d'une mobilisation partielle de cette épargne pour financer des projets capables de créer un surplus, c'est-à-dire des projets dans le secteur formel: assurer, en somme, un décloisonnement des circuits de financement au profit des entreprises du secteur moderne, pour reprendre la terminologie appropriée. Cette opération aurait pour effet également de mieux enraciner le secteur moderne dans l'ensemble de la société, évitant ainsi qu'il ne se constitue comme une enclave extravertie financée, approvisionnée et tournée vers l'extérieur de façon trop exclusive.

17. G. Nihan, « Le secteur non structuré, signification, aire d'extension du concept et application expérimentale », Tiers Monde, 1980, ${ }^{\circ} 82$.

18. C. Moser et J. Marsie Hasen, «A survey of empirical studies in industrial and manufacturing activities in the informal sector in the developing countries », UNIDO, 1984. 